\title{
Automated Voxel Placement: A Linux-based Suite of Tools for Accurate and Reliable Single Voxel Coregistration
}

Eric A. Woodcock", Muzamil Arshad, Dalal Khatib and Jeffrey A. Stanley

Brain Imaging Research Division, Department of Psychiatry and Behavioral Neurosciences, Wayne State University School of Medicine, Detroit, MI 48201, USA

\section{'Correspondence to:}

Eric A. Woodcock, PhD

Department of Psychiatry and Behavioral

Neurosciences, Tolan Park Medical Building

3901 Chrysler Service Drive, Suite 2A

Detroit, MI 48201, USA

Tel: +13135778257

Fax +1 3135775900

E-mail: ewoodcoc@med.wayne.edu

Received: October 26, 2017

Accepted: February 05, 2018

Published: February 08, 2018

Citation: Woodcock EA, Arshad M, Khatib D, Stanley JA. 2018. Automated Voxel Placement: A Linux-based Suite of Tools for Accurate and Reliable Single Voxel Coregistration. $J$ Neuroimaging Psychiatry Neurol 3(1): 1-8.

Copyright: (c) 2018 Woodcock et al. This is an Open Access article distributed under the terms of the Creative Commons Attribution 4.0 International License (CC-BY) (http:// creativecommons.org/licenses/by/4.0/) which permits commercial use, including reproduction, adaptation, and distribution of the article provided the original author and source are credited.

Published by United Scientific Group

\begin{abstract}
Background: Single-voxel proton magnetic resonance spectroscopy $\left({ }^{1} \mathrm{H}\right.$ MRS) is a powerful technique for studying in vivo neurochemistry, but has an often-overlooked source of error variance: inconsistent voxel placement between scans. We developed and evaluated an Automated Voxel Placement (AVP) procedure for accurate and reliable ${ }^{1} \mathrm{H}$ MRS voxel prescription. AVP is a suite of Linux-based programs that facilitate automated template-driven single-voxel coregistration.
\end{abstract}

Methods: Three studies were conducted to evaluate AVP for prescription of one voxel: left dorsolateral prefrontal cortex. First, we evaluated how robust AVP was to 'extreme' subject head positions/angulations within the scanner head coil. Second, subjects $(\mathrm{N}=13)$ were recruited and underwent MR scans. Manual voxel prescription $(\mathrm{n}=5)$ was contrasted with AVP $(\mathrm{n}=8)$. A subset of AVP subjects $(n=4)$ completed a second scan. Third, ongoing data collection $(n=16$; recruited for a separate study) helped evaluate AVP. Voxel placement accuracy was quantified as $3 \mathrm{D}$ geometric voxel overlap percentage between each subject's voxel and the template voxel. Reliability was quantified as $3 \mathrm{D}$ geometric voxel overlap percentage across subjects at each time point and within subjects who completed two scans.

Results: Results demonstrated that AVP was robust to 'extreme' head positions (97.5\% - 97.9\% overlap with the template voxel). AVP was significantly more accurate (baseline and follow-up: $96.2 \% \pm 3.0 \%$ and $97.6 \% \pm 1.4 \%$ overlap) than manual voxel placement $(67.7 \% \pm 22.8 \%$ overlap; $p s<.05)$. AVP was reliable within- (97.9\%) and between-subjects $(94.2 \%$ and $97.2 \%$ overlap; baseline and follow-up; respectively). Finally, ongoing data collection indicates AVP is accurate (96.0\%).

Conclusion: These pilot studies demonstrated that AVP was feasible, accurate, and reliable method for automated single voxel coregistration.

\section{Keywords}

Magnetic resonance spectroscopy, Single voxel, Automated voxel placement, Coregistration, Neurochemistry

\section{Introduction}

In vivo proton magnetic resonance spectroscopy ( ${ }^{1} \mathrm{H}$ MRS) is a powerful non-invasive neuroimaging technique that can quantify neurochemical levels in localized brain areas [1-3]. For example, ${ }^{1} \mathrm{H}$ MRS can distinguish tumor from healthy brain tissue $[4,5]$, locate tissue affected by seizure disorders [69], and identify cerebral atrophy associated with dementia and mild cognitive 
impairment [10-16]. In addition, ${ }^{1} \mathrm{H}$ MRS has been used to track disease progression $[14,17,18]$ and treatment response over time within subjects $[19,20]$.

Single-voxel ${ }^{1} \mathrm{H}$ MRS (SVS) facilitates measurement of biochemical information in a three-dimensional volume (voxel) located in a specific anatomical region. However, inconsistent and unreliable voxel placement between subjects in crosssectional studies (i.e., to contrast neurological/psychiatric diagnoses or medication responses) or within subjects across time in longitudinal studies (i.e., treatment response) is a source of error variance that is often overlooked in SVS studies $[21,22]$. It is well-established that neurochemistry varies by tissue type (grey matter vs. white matter vs. cerebrospinal fluid) and anatomical location [23, 24]. Indeed, prior research has demonstrated that metabolite levels differed by up to $30 \%$ depending on voxel placement; thus, highlighting the importance of accurate and reliable voxel placement for SVS [25-27]. Unfortunately, SVS studies often rely on manual voxel prescription by an operator for each research subject based on (subjective) anatomical features. This process can be time-consuming, inconsistent, and challenging. Moreover, SVS studies often do not report voxel placement reliability and ignore the error variance contributed by inconsistent voxel placement. In the few published studies that reported percentage of 3D geometric voxel overlap between subjects, overlap was $\sim 70 \%$ [28] or worse [29].

Several techniques have been developed to address the problem of voxel placement inconsistency, including methods by Hancu et al. and Storrs et al. The Hancu method [30, 31] used a registration algorithm to automatically reposition the manually-placed voxel from the subject's first scan. This approach was highly-reliable (94\% - 95\% within-subject voxel overlap). However, this approach required manual placement for each subject's first scan: thus, limiting its utility in research settings $[30,31]$. At the time of publication, the authors were able to find one automated method capable of between-subject voxel coregistration: the Storrs method [29].The Storrs method used a template-drive and demonstrated excellent withinsubject voxel replacement reliability: 97\% - 98\% (depending on anatomical location), but modest between-subject median voxel placement accuracy (overlap with template voxel): $81 \%$ $84 \%$ (depending on anatomical location) [29]. Voxel overlap shared across subjects (i.e., between-subject reliability) was not reported for the Storrs method. Inconsistent and unreliable voxel placement between subjects remains a significant obstacle for SVS research studies.

The aim of the present study was to develop and evaluate an automated voxel placement technique for reliable voxel coregistration within- and between-subjects. Automated Voxel Placement (AVP) is a suite of Linux-based computer programs/ scripts designed to promote implementation using existing coregistration algorithms. An a priori voxel location in the left dorsolateral prefrontal cortex (dlPFC) was selected (challenging location for manual voxel placement) and evaluated. Two studies evaluated the AVP method. First, we conducted a 'proof-ofprinciple' study to evaluate how robust AVP coregistration was to 'extreme' subject head positions within the head coil.
Second, a sample of research subjects $(\mathrm{N}=13)$ were recruited to evaluate the efficacy of the AVP approach $(n=8)$ vs. manual placement $(n=5)$. We hypothesized that the AVP approach would be significantly more accurate and reliable than the most commonly used approach: manual voxel placement.

\section{Materials and Methods}

\section{Participants}

All procedures performed in studies involving human participants were in accordance with the ethical standards of the institutional and/or national research committee and with the 1964 Helsinki declaration and its later amendments or comparable ethical standards. Thirteen volunteers were recruited locally to participate in this research study and were compensated for their time. Informed consent was obtained from all individual participants included in the study. All 13 subjects completed the baseline MRI scan. Manual voxel prescription was used for five subjects, while AVP was used for the remaining eight subjects. In addition, a subset of AVP participants $(n=4)$ completed a follow-up MRI scan $~ 2.5$ days later (on average) to evaluate AVP's within-subject reliability.

An experienced MR technologist (DK) used a 2D printout that depicted the optimal voxel location in template brain space along three orthonormal directions (sagittal, coronal, and axial). The voxel was prescribed using a wireframe depiction of the voxel location on a computer system display along three orthonormal directions. The Principal Investigator (EW) confirmed each voxel placement before it was finalized. This procedure was replicated for all five subjects who received manual voxel prescription.

\section{AVP suite}

The AVP suite is available free-of-charge: https:// github.com/ewoodcock/avp_scripts.git. AVP was developed and evaluated using an adult template brain ('LAS' image orientation) on a 3 Tesla Siemens Verio system. However, the AVP method was designed to be scanner system-, field strength-, subject population-, and anatomy-independent. Three programs are included in the AVP suite: 'AVP_Create', 'AVP_Coregister', and 'AVP_Overlap.'

\section{AVP_Create}

A schematic diagram of the processing logic used in 'AVP_Create is depicted in figure 1. Upon execution of the 'AVP_Create' script, the user will be prompted to enter voxel parameters, including: voxel location (center coordinate), voxel dimensions, voxel angulation/rotation about each axis $[\mathrm{T}>\mathrm{S}$ $(\mathrm{x}), \mathrm{T}>\mathrm{C}(\mathrm{y})$, Rotation $(\mathrm{z})$ ], voxel description and study name, and image orientation (e.g. 'LAS'). 'AVP_Create' will generate the specified volume and prompt the user to visually appraise it in the template image space using the $3 \mathrm{D}$ viewer (FSL view; FMRIB, Oxford, UK). If the voxel position is inadequate, the user can make the necessary adjustments. This is an iterative process and will likely require several adjustments $(<1 \mathrm{~min}$ computer processing time per adjustment). Once the voxel position is optimal, all voxel information is stored in the 'voxel_ 
locations.txt' file for future coregistration. Additional voxel locations can be created using 'AVP_Create' by repeating this process. In this way, the user can generate a library of studyspecific template voxels (each with a study-specific template image) that are retained for future coregistration.

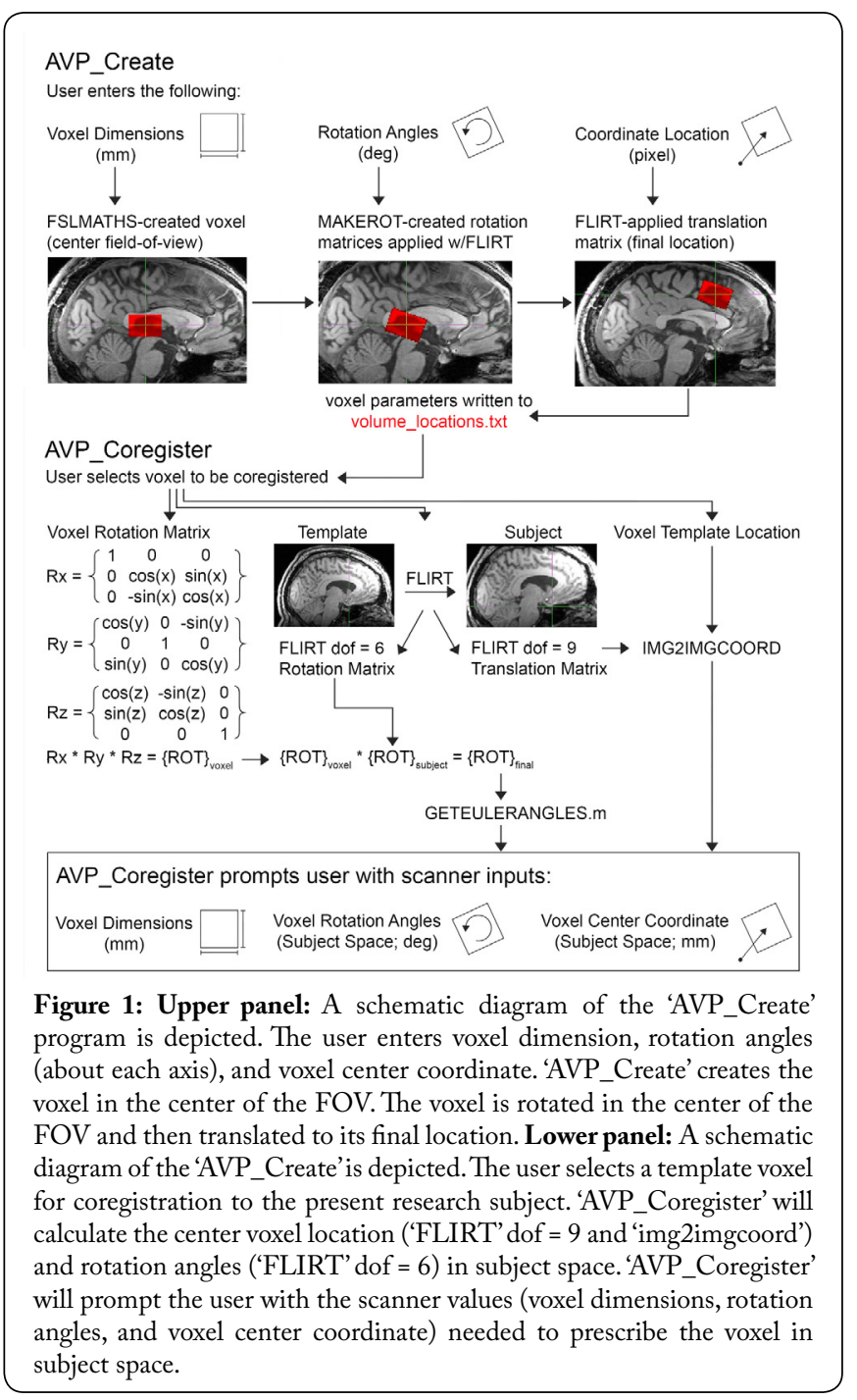

\section{AVP_Coregister}

Upon execution of the 'AVP_Coregister' script, the user must input the study name, subject ID, and time point for the current research subject (Figure 1), and select the template voxel for coregistration. The template anatomical image was coregistered (rigid; 6 degrees of freedom [dof]; 'FLIRT' [32]) to the subject $\mathrm{T}_{1}$-weighted anatomical image. The default cost function (adopted herein) used by 'FLIRT' is Correlation Ratio with trilinear interpolation. 'FLIRT' combines a local and global search strategy to optimize coregistration. The rigid-body coregistration matrix (linear translation and rotation adjustments along all three axes) was inverted and used to calculate Euler rotation angles. A second linear (affine) coregistration (dof = 9; 'FLIRT') and 'img2imgcoord' function was used to calculate voxel center coordinates in subject space. Nine dof limited 'FLIRT' to linear translation, rotation, and scaling adjustments along all three axes to coregister the images. Finally, those parameters (voxel dimensions, center coordinate, and rotation angles) were input at the scanner. The authors recommend visual appraisal to confirm the voxel placement is optimal.

\section{AVP_Overlap}

Upon execution of 'AVP_Overlap', voxel attributes were extracted from the dicom file header information for each subject at each time point. Thus, voxel overlap was calculated based on the region from which MRS spectra were acquired in each subject, and not values or transformation matrices calculated by 'AVP_Create' or 'AVP_Coregister'. The processing pipeline for 'AVP_Overlap' mirrored 'AVP_Coregister' and used two coregistration procedures (rigid-body dof = 6 'FLIRT' for calculation of Euler angles [rotation matrix] and linear dof $=9$ 'FLIRT' for calculation of voxel coordinate location [translation matrix]). Next, the voxel was 'reconstructed' in template space using the prescribed voxel dimensions. This facilitated accurate calculation of rotation angles and voxel center coordinate that is calibrated for anatomical size differences (between the template and subject anatomy) while preserving voxel dimensions. Finally, the voxel is binarized: every pixel location has a value between 0 (voxel not present) and 1 (voxel present). It was important to consider the 'partial volume' problem. Any voxel that is defined as a binary mask (ones and zeroes) and is rotated/angulated with respect to the Field-of-View (FOV) will have pixels along the edges with pixel intensity values somewhere between 0 and 1 due to the resampling of the voxel mask (Figure 2; upper panel). To minimize the impact of this effect on the accuracy of voxel overlap calculation, two approaches were implemented. First, the entire matrix was resampled at a pixel resolution of $0.5 \mathrm{~mm}$ isotropic to minimize partial volume effects. Second, a range of pixel intensity thresholds are calculated in the 'AVP_Overlap' script. The user should select the pixel intensity threshold closest to, without exceeding, $100 \%$ of the unrotated template voxel size (labeled 'Percent Total Voxel' in the 'Overlap_Summary. txt' file) for all voxel overlap metrics. If the template voxel is not rotated (i.e., orthogonal to the FOV), the user should select the highest pixel intensity threshold provided (0.95).

'AVP_Overlap' automatically calculates several metrics for evaluation of voxel placement. 1) Voxel Placement 'Accuracy'. This is defined as the percentage of $3 \mathrm{D}$ geometric voxel overlap between a subject's voxel and the template voxel. The binarized subject voxel is added to the binarized template voxel in template space such that pixel values sum. Next, the average pixel value in every pixel location is calculated (Figure 2; lower panel). The number of pixels from the averaged image that exceed the pixel intensity threshold are quantified and divided by the number of pixels in the template voxel (in isolation) that exceed the pixel intensity threshold. This process is repeated separately for every subject voxel before a group mean is calculated and expressed as a percentage. If more than one experimental time point exists (i.e., longitudinal study), this process is repeated separately for each time point. 2) BetweenSubject Overlap. Every binarized subject voxel is summed in template space and an average pixel value is quantified for every pixel location. Again, the number of pixels that exceed 


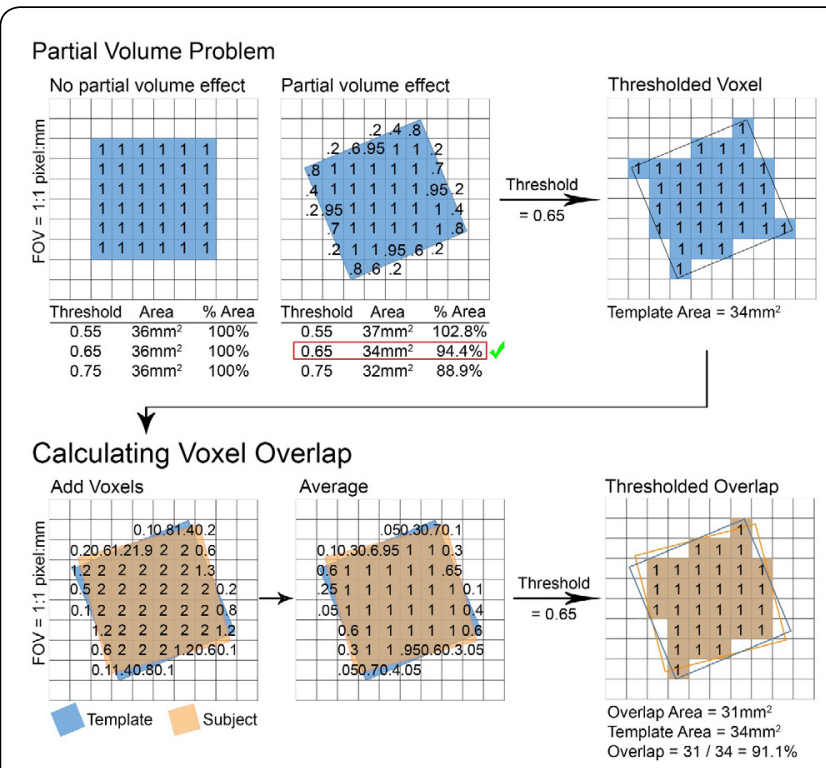

Figure 2: Upper panel: A schematic representation of the 'partial volume problem' is depicted. On the left, a blue $6 \mathrm{~mm} \times 6 \mathrm{~mm} 2 \mathrm{D}$ voxel is orthogonal to the Field-of-View (FOV; i.e., unrotated). The voxel occupies $100 \%$ of the $2 \mathrm{D}$ pixel space in every pixel location, and thus, every location has a pixel intensity value $=1\left(\right.$ area $\left.=36 \mathrm{~mm}^{2}\right)$ at all pixel intensity threshold levels (i.e., no partial volume effect). In the upper middle panel, the $2 \mathrm{D}$ voxel is rotated with respect to the FOV, thus creating a 'partial volume effect.' Along the edges of the voxel, pixel intensity values range between 0 and 1 . Thus, depending on the pixel intensity threshold (i.e. the threshold beyond which the voxel is deemed present [vs. not present] in each pixel location), the voxel area will range between $37 \mathrm{~mm}^{2}$ (102.8\% of 'true' template voxel area; i.e., its area when orthogonal to the FOV or $36 \mathrm{~mm}^{2}$ ) and $32 \mathrm{~mm}^{2}(88.9 \%)$. The user should select the pixel intensity threshold that corresponds to closest to, without exceeding, $100 \%$ of the unrotated area (i.e., 36 $\mathrm{mm}^{2}$ ). The appropriate pixel threshold is 0.65 which corresponds to a thresholded voxel area of $34 \mathrm{~mm}^{2}$ (figure on the upper right) or $94.4 \%$ of the template area (i.e., $34 \mathrm{~mm}^{2} / 36 \mathrm{~mm}^{2}$ ). The user should report voxel overlap results that correspond to the selected pixel intensity threshold. Lower panel: The analytic strategy for calculating voxel overlap with the template voxel (i.e., voxel placement 'accuracy') is depicted. The binarized subject voxel is added to the binarized template voxel in template space (after coregistration using 'AVP_Overlap') such that pixel values sum. At every pixel location, an average pixel value is calculated and thresholded (at the selected pixel intensity threshold: 0.65 ). The overlap area is quantified and expressed as a percentage of the template area (using the same pixel intensity threshold: 0.65 ). In this example, the thresholded overlap area is $31 \mathrm{~mm}^{2}$, which is divided by the template area $\left(34 \mathrm{~mm}^{2}\right)$, and expressed as a percentage: $91.1 \%$ voxel overlap. This example depicts calculation of voxel placement accuracy: subject voxel overlap percentage with the template voxel. However, the same steps are repeated for between-subject overlap and within-subject overlap, with one difference - only subject voxels are used for those metrics. The template voxel is not included in the calculation of between- or withinsubject overlap, only voxel placement 'accuracy'.

the pixel intensity threshold from the averaged image is divided by the number of pixels in the template voxel (in isolation) that exceed that threshold and is expressed as a percentage. This process is repeated separately for each experimental time point. 3) Within-Subject Overlap. If multiple experimental time points exist, voxel overlap across time points is calculated separately for each subject. Binarized subject voxels from each time point are summed, pixel values averaged, and then thresholded (again, using the pixel intensity threshold). Next, summary statistics are calculated across subjects: group mean $\%$ within-subject voxel overlap and coefficient of variation (CV\%). In addition, partial volume tissue segmentation maps (using 'FAST'; estimated from $\mathrm{T}_{1}$-weighted scan) within the voxel space are calculated for each subject. Group mean percentage of gray and white matter and cerebrospinal fluid are reported for each experimental time point.

The authors note that RF pulses are imperfect (especially $180^{\circ}$ RF pulses; i.e., PRESS), and thus, are associated with rounded MRS voxel edges. However, for purposes of geometric voxel overlap calculation, all voxels were assumed to be perfectly rectangular with $90^{\circ}$ angles and flat edges.

\section{AVP computer processing}

'AVP_Create' and 'AVP_Coregister' were executed on a laptop computer with an Intel Core i7 $2.60 \mathrm{GHz}$ processer and 64-bit OS running Windows version 8.1 (Microsoft Corporation, Redmond, WA). The scripts were executed in an Oracle Linux Virtual Machine (Oracle Corporation, Redwood Shores, CA; 4 GB of dedicated RAM). Computer processing time for 'AVP_Create' and 'AVP_Coregister' were acceptable ( $<1$ minute and $\sim 2$ minutes, respectively).

\section{Neuroimaging}

All imaging was conducted on a 3 Tesla Siemens Verio system with a 32-channel receive-only volume head coil. High resolution $\mathrm{T}_{1}$-weighted structural scans were collected using the 3D Magnetization Prepared Rapid Gradient Echo (MPRAGE) sequence with the following parameters: $\mathrm{TR}=$ $2.2 \mathrm{~s}, \mathrm{TE}=3 \mathrm{~ms}, \mathrm{~T}_{\mathrm{I}}=799 \mathrm{~ms}$, flip angle $=13^{\circ}, \mathrm{FOV}=256 \times 256$ x $160 \mathrm{~mm}, 256 \times 1 \mathrm{~mm}$ thick axial slices, matrix = $176 \times 256$.

\section{Voxel placement}

The voxel location evaluated in this study is depicted in figure 3. Voxel dimensions were $1.5 \times 2.0 \times 1.5 \mathrm{~cm}^{3}$ (volume = $4.5 \mathrm{~cm}^{3}$ ). The voxel was located in the left dlPFC (Brodmann Areas 45 and 46). The dlPFC was selected because it is a challenging location for consistent manual voxel placement.

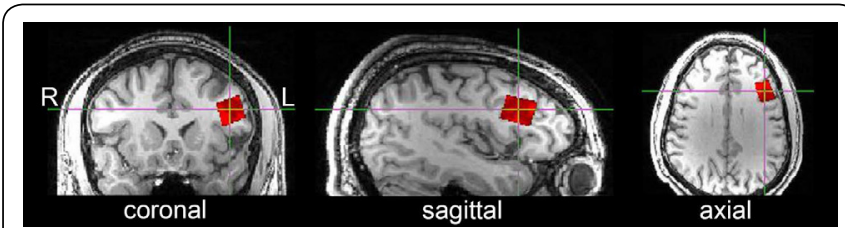

Figure 3: The voxel investigated in this study is depicted in orthonormal slices. The voxel is located primarily in Brodmann Areas 45 and 46 (dlPFC; 15 × $20 \times 15 \mathrm{~mm}$ ).

\section{Proof-of-principle}

As a 'proof-of-principle' evaluation, we evaluated three 'extreme' head positions for a single subject (Figure 4) to evaluate how robust AVP was to subject head position in the head coil. The following protocol was repeated three times during a single scanning session ( 45 min): 1) the technologist positioned the subject in the scanner, 2) $\mathrm{T}_{1}$-weighted structural images were collected (using MPRAGE), and 3) the template voxel (Figure 3; left dlPFC) was coregistered and prescribed (using 'AVP_Coregister'). Three different analyses were conducted to evaluate the reliability of 'AVP_Coregister' for this 'proof-of-principle' investigation. First, percentage 
of 3D geometric voxel overlap between each of the three voxel placements and the template voxel was calculated (i.e. 'accuracy'). Second, percentage of 3D geometric overlap and CV\% across the three voxels was calculated (i.e. 'reliability'). Third, voxel tissue composition was calculated and evaluated for consistency $(\mathrm{CV} \%)$.

\section{Ongoing data collection}

In subsequent research studies conducted in our laboratory, the identical left dIPFC voxel was prescribed using AVP. Thus, to evaluate the reliability of AVP in a larger sample, we included an additional 16 subjects ( 24 subjects in total) and evaluated between-subject voxel overlap.

\section{Analysis strategy}

Results were calculated using the 'AVP_Overlap' script. The primary outcome variables for this study were: 1 ) voxel placement 'accuracy', 2) between-subject voxel overlap (i.e., 'reliability'), and 3) within-subject voxel overlap, each of which was defined above (see 'AVP_Overlap'). For the present voxel, a 0.65 pixel threshold was selected $(97.2 \%-98.7 \%$ of the unrotated template voxel size). We report mean percentage of voxel placement accuracy and between-subject voxel overlap separately for each timepoint: baseline and followup. For within-subject overlap, we report mean percentage of within-subject overlap and CV\% (for subjects who completed both scans). In addition, using the partial volume tissue segmentation maps within the voxel space, we calculated CV\% between baseline and follow-up scans for gray and white matter (i.e., 'within-subject anatomical consistency').

Results using manual voxel placement $(n=5)$ were contrasted with the AVP approach at baseline $(n=8)$ and follow-up ( $n=4)$ using one-way analyses of variance. Descriptive statistics are presented as mean $(\mathrm{M}) \pm$ one standard deviation (SD; unless otherwise noted).

\section{Results}

\section{Proof-of-principle}

Prior to subject recruitment or enrollment, AVP was evaluated on a single subject during a single scanning session using three different 'extreme' head positions in the head coil (Figure 4). Table 1 describes the translational and rotational differences for each of the three head positions relative to the template voxel. 3D geometric voxel overlap between each head position and the template voxel demonstrated that AVP was highly accurate (median: 97.7\%; range: 97.5\%-97.9\% overlap). Mean 3D geometric voxel overlap across all three head positions demonstrated AVP was highly reliable $(98.9 \%$ overlap) and consistent $(\mathrm{CV} \%=0.19 \%)$. Tissue segmentation results demonstrate that anatomical composition across the three head positions was consistent (grey matter CV\% = $1.63 \%$, range $=24.8 \%-25.8 \%$; white matter CV\% $=0.84 \%$, range $=72.9 \%-74.4 \%)$. Together, these data demonstrated that the AVP approach was robust to subject head position in the head coil.

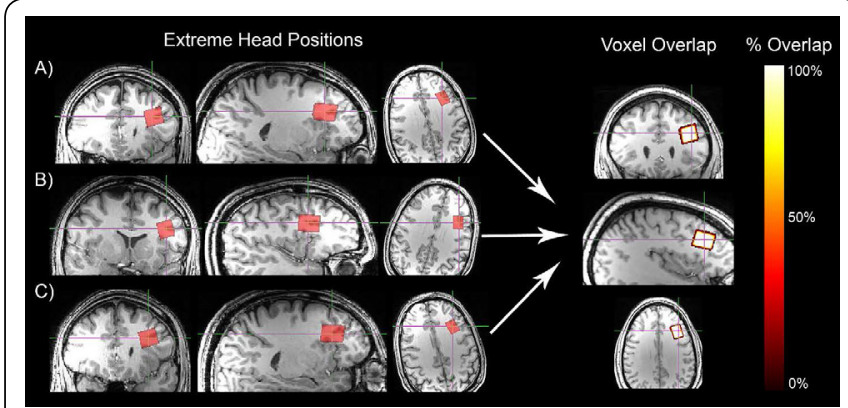

Figure 4: Left panel: Orthonormal slices of the three extreme head positions evaluated during the 'proof-of-principle' investigation is depicted. Right panel: Using voxel parameter information from the dicom file generated during ${ }^{1} \mathrm{H}$ MRS acquisition, each voxel was recreated in subject space, coregistered to template space, and $3 \mathrm{D}$ geometric voxel overlap was evaluated. Note: white indicates complete voxel overlap, yellow-orange gradient indicates incomplete voxel overlap, and red indicates no voxel overlap.

Table 1: Extreme head positions.

\begin{tabular}{|l|l|l|l|l|l|l|}
\hline & \multicolumn{3}{|l|}{ Voxel Center Coordinate $(\mathrm{mm})$} & \multicolumn{3}{l|}{ Rotation Angles (deg) } \\
\hline & $\mathrm{X}(\mathrm{L}-\mathrm{R})$ & $\mathrm{Y}(\mathrm{A}-\mathrm{P})$ & $\mathrm{Z}(\mathrm{F}-\mathrm{H})$ & $\mathrm{T}>\mathrm{S}$ & $\mathrm{T}>\mathrm{C}$ & Rotation \\
\hline Template & 32 & 25 & 22.5 & 7.0 & 20.0 & 15.0 \\
\hline Extreme A & 23 & 20 & 35 & 16.9 & 5.8 & 25.3 \\
\hline Extreme B & 50 & -1.4 & 27 & 15.9 & 3.3 & -1.7 \\
\hline Extreme C & 19 & 13 & 32 & 15.8 & 1.7 & 27.4 \\
\hline & $\Delta$ Translation $(\mathrm{mm})$ & & \multicolumn{4}{|l|}{$\Delta$ Rotation (deg) } \\
\hline Template to A & -9 & -5 & 12.5 & 9.9 & -14.2 & 10.3 \\
\hline Template to B & 18 & -26.4 & 4.5 & 8.9 & -16.7 & -16.7 \\
\hline Template to C & -13 & -12 & 9.5 & 8.8 & -18.3 & 12.4 \\
\hline
\end{tabular}

Note: The voxel center coordinate and rotation angles for each of the 'extreme' head position scans in subject space are depicted in the upper rows. The translational and rotational differences for each voxel relative to the template are depicted in the lower rows.

\section{Participant characteristics}

Thirteen subjects were recruited for formal evaluation of AVP. The modal participant was a 28 -year-old (mean \pm 1 SD: $27.7 \pm 3.8$ yrs old; range: $21-34$ yrs) African-American (76.9\%) male (84.6\%).

\section{Voxel placement accuracy}

AVP was more accurate than manual voxel placement $(\mathrm{n}=$ $5)$ at baseline $(\mathrm{n}=8)$ and follow-up $(\mathrm{n}=4): F(1,12)=12.84$, $p<.005$ and $F(1,8)=6.76, p<.05$, respectively (Figure 5). Mean accuracy $( \pm 1 \mathrm{SD})$ using AVP was higher $(96.2 \% \pm 3.0 \%$ and $97.6 \% \pm 1.4 \%$; baseline and follow-up, respectively) than manual voxel placement $(67.7 \% \pm 22.8 \%)$.

\section{Within-subject reliability}

A subset of participants in the AVP condition returned for a follow-up visit $(n=4)$. Mean within-subject overlap using AVP was $97.3 \% \pm 2.1 \%$. Mean gray and white matter percentage of voxel composition were: $32.2 \%$ (range: $20.4 \%-$ $38.3 \% ; \mathrm{CV} \%=10.8 \%$ ) and $65.5 \%$ (range: $58.5 \%-79.5 \%$; CV\% $=5.2 \%)$, respectively.

One outlier for gray matter $(\mathrm{CV} \%=28.3 \%$; all other values 
$<10 \%)$ was observed. This value was associated with relatively poor within-subject reliability (93.8\% overlap). In this subject, percentage of voxel composed of gray matter was estimated to be $36.5 \%$ at baseline and $20.4 \%$ at follow-up. This outlier illustrated that small voxel coregistration errors can influence voxel tissue composition, which, in turn, will bias estimates of neurochemistry.

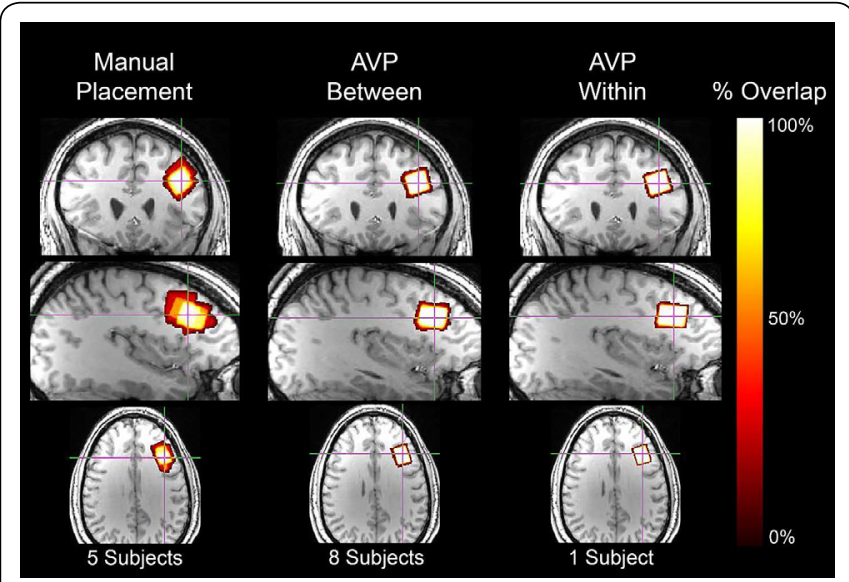

Figure 5: Orthonormal slices of 3D geometric voxel overlap in template space are depicted. Left panel: Voxel overlap across subjects using manual voxel placement $(n=5)$ is depicted. Center panel: Voxel overlap across subjects using AVP ( $\mathrm{n}=8$; baseline) is depicted. Right panel: Voxel overlap within a single subject across timepoints using AVP is depicted. Note: white indicates complete voxel overlap, yellow-orange gradient indicates incomplete voxel overlap, and red indicates no voxel overlap.

\section{Between-subject reliability}

Manual voxel placement resulted in poor between-subject reliability: $67.7 \%$. However, this value was consistent with the literature for manual voxel placement ( $70 \%$ between-subject voxel overlap) [28, 33, 34]. Mean between-subject overlap using AVP was $94.2 \%$ at baseline and $97.2 \%$ at follow-up.

\section{Ongoing data collection}

To date, AVP has been used to prescribe this identical left dlPFC voxel in 24 subjects in subsequent research studies in our laboratory. Results indicated 3D geometric voxel overlap percentage (between-subject reliability) was excellent across 24 subjects (96.0\%; Figure 6).

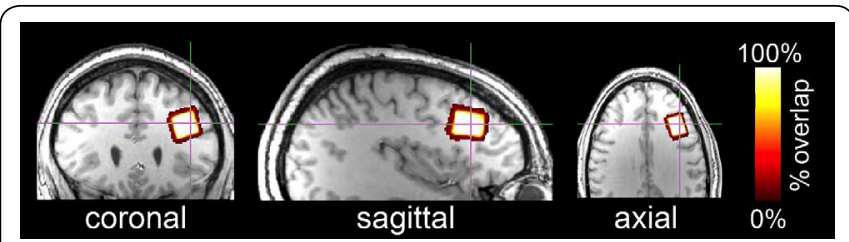

Figure 6: Orthonormal slices of 3D geometric voxel overlap $(n=24)$ in template space are depicted.

\section{Discussion}

In this study, we evaluated a method for automated voxel placement using a prospective, template-driven approach. The AVP suite facilitated creation of template voxel positions that could be automatically coregistered and prescribed at the scanner $(\sim 2$ min computer processing time). Results indicate AVP resulted in highly accurate and reliable voxel coregistration. AVP used existing coregistration algorithms in a novel and freely-available package designed to promote implementation, especially in clinical SVS research studies.

Results from this study demonstrated that AVP was feasible and accurate. 'AVP_Create' facilitated creation of a library of template voxels that were retained for future coregistration. A different template image can be designated for each research study, subject population, and anatomical region of interest. Prior to subject scanning, users appraise and iteratively adjust each template voxel location (using a $3 \mathrm{D}$ viewer) until optimal. This process saved valuable scan time and facilitated accurate template voxel placement (less error prone than manual voxel placement guided by 2D anatomical images). At the scanner, 'AVP_Coregister' facilitated accurate voxel prescription based on the subject's $\mathrm{T}_{1}$-weighted anatomical scan. A rigid-body $($ dof $=6)$ coregistration procedure was used to calculate Euler rotation angles, while a linear procedure $($ dof $=9)$ was used to calculate voxel center coordinates in subject space. This approach provided an optimal balance between efficiency (computer processing time $=\sim 2$ minutes) and accuracy. Mean $3 \mathrm{D}$ geometric voxel overlap between each subject voxel and the template voxel (i.e. voxel placement 'accuracy') was $96.2 \%$ at baseline and $97.6 \%$ at follow-up. Importantly, overlap was calculated based on the actual voxel location from which spectra were acquired (parameters extracted from each subject's dicom header file) and not matrices or coordinates generated by AVP. These data demonstrated that the median subject voxel placement was inaccurate approximately $0.15 \mathrm{~mm}$ in a given direction relative to the template voxel (likely due to rounding and partial volume effects). At this level of accuracy, it is likely that subject motion will contribute greater voxel placement error. In contrast, manual voxel placement yielded mean overlap accuracy of $67.7 \%(\sim 2 \mathrm{~mm}$ placement error in a given direction; comparable to prior research $[28,33])$. Finally, AVP was substantially more accurate voxel coregistration than the most accurate published approach known to the authors $(\sim 13 \%$ overlap improvement; Cohen's d $\geq 2.3$ [large effect]) [29].

In addition to placement accuracy, we calculated withinsubject voxel overlap reliability and voxel tissue composition consistency across the two scans ( 2.5 days apart). Withinsubject AVP coregistration across subjects was highly reliable and consistent (mean: 97.3\%). These data indicated that mean voxel replacement inaccuracy was approximately $0.2 \mathrm{~mm}$ in any given direction. AVP was roughly equivalent to the most reliable published approach [29]. Voxel tissue composition (percentage of grey vs. white matter) was consistent within individuals across scanning sessions and during the 'extreme' head position 'proof-of-principle' test.

Finally, we calculated between-subject voxel overlap (mean voxel space shared across subjects in template space). Our highly-experienced SVS research team demonstrated poor between-subject reliability using manual voxel prescription guided by $2 \mathrm{D}$ anatomical images (overlap: $67.7 \%$ ). In contrast, AVP was highly reliable and consistent at each time point (mean overlap: 94.2\% and 97.2\%; baseline and follow-up, respectively). Between-subject reliability was not reported for the Storrs method [29]. 
There exist other sophisticated automated coregistration approaches in the literature. Van der Kouwe et al. developed an automated real-time (while the subject is in the scanner) procedure in which they collect two medium resolution, large FOV images (two different tissue contrasts) and use a rigidbody transformation matrix (similar to the procedure described herein) to coregister each subject to a pre-determined template brain [35]. This procedure offers similar advantages (real-time coregistration to a template brain) as our approach. It is difficult to compare this approach with the AVP method because the authors did not evaluate voxel overlap. However, we believe the AVP method facilitated superior voxel placement reliability. Van der Kouwe and colleagues found that within-subject whole-brain test-retest reliability was significantly improved by a post-scan 'FLIRT,' which indicated the presence of error for within-subject coregistration [35]. AVP does not benefit from a post-scan 'FLIRT' for either within-subject or between-subject voxel replacement.

The authors were aware of other automated voxel placement methods [34, 36-40]. These approaches were not discussed in this paper for two reasons: 1) similar to others described in this manuscript, and/or 2) notable limitations existed relative to approaches described herein (e.g. more time-consuming less reliable, etc.). This study had several limitations. This study included a small number of research subjects (though subsequent data collection indicated high reliability across 24 subjects) and only one voxel location. However, AVP demonstrated robust and statistically-significant superiority to manual voxel placement. AVP has not been tested on subjects with significant morphological changes (e.g., brain atrophy, tumor, or injury), but the authors presume accuracy/reliability would be impaired due to coregistration imperfections between the subject's brain and the template.

\section{Conclusion}

In summary, single voxel MRS research studies suffer from an often-overlooked source of error variance: inconsistent voxel placement. To combat this problem, we developed an approach that leveraged existing coregistration algorithms in a user-friendly package to facilitate automated template-driven voxel prescription and post-scan voxel overlap calculation. Our results demonstrated that AVP was significantly more accurate and reliable than the most commonly-used voxel placement method (manual prescription guided by anatomical images) and the most accurate published method (Storrs [29]) known to the authors. AVP can be easily implemented across scanner platforms (though not currently compatible with General Electric systems), field-strength, subject population, anatomy, and is available free-of-charge: https://github.com/ ewoodcock/avp_scripts.git.

\section{Funding}

Research reported in this publication was supported by the National Institute on Drug Abuse of the National Institutes of Health under Award Number F31 DA040369 (awarded to Woodcock). The content is solely the responsibility of the authors and does not necessarily represent the official views of the National Institutes of Health. Funding also provided by Wayne State University (New Investigator Grant; awarded to Woodcock), State of Michigan (Joe Young Sr./Helene Lycaki funds; Stanley), and the Detroit Wayne Mental Health Authority (Stanley). Funding sources were not involved in the design, execution, analysis or interpretation of these data.

\section{Acknowledgements}

The authors thank Caroline Zajac-Benitez,Michael Lisieski, Natalie Wiseman, Brian Silverstein, Vikas Kodali, Andrew Neff, Chaitali Anand, and Wafaa Sweidan for their assistance.

\section{Authors' Contribution}

The inspiration of AVP was provided by Stanley. Woodcock developed the approach and protocol, scripted the AVP suite, collected the data, and authored the manuscript. Arshad provided invaluable assistance in the development/ evaluation of AVP and edited the manuscript. Khatib operated the MRI scanner and assisted with data collection. Stanley oversaw development of AVP and data collection, as well as edited the manuscript. All authors have read and approved of this manuscript.

\section{Conflicts of Interest}

The authors declare that they have no conflict of interest.

\section{Research Involving Human Participants}

All procedures performed in studies involving human participants were in accordance with the ethical standards of the institutional and/or national research committee and with the 1964 Helsinki declaration and its later amendments or comparable ethical standards.

\section{Informed Consent}

Informed consent was obtained from all individual participants included in the study.

\section{References}

1. Keshavan MS, Stanley JA, Pettegrew JW. 2000. Magnetic resonance spectroscopy in schizophrenia: methodological issues and findingspart II. Biol psychiatry 48(5): 369-380. https://doi.org/10.1016/S0006 3223(00)00940-9

2. Stanley JA. 2002. In vivo magnetic resonance spectroscopy and its application to neuropsychiatric disorders. Can J Psychiatry 47(4): 315326. https://doi.org/10.1177/070674370204700402

3. Stanley JA, Pettegrew JW, Keshavan MS. 2000. Magnetic resonance spectroscopy in schizophrenia: methodological issues and findingspart I. Biol psychiatry 48(5): 357-368. https://doi.org/10.1016/S00063223(00)00949-5

4. Alger J, Frank J, Bizzi A, Fulham M, DeSouza B, et al. 1990. Metabolism of human gliomas: assessment with H-1 MR spectroscopy and F-18 fluorodeoxyglucose PET. Radiology 177(3): 633-641. https:// doi.org/10.1148/radiology.177.3.2243962

5. Bruhn H, Michaelis T, Merboldt K, Hänicke W, Gyngell M, et al.1992. On the interpretation of proton NMR spectra from brain tumours in vivo and in vitro. NMR Biomed 5(5):253-258. https://doi.org/10.1002/ nbm. 1940050510 
6. Bruton CJ. 1988. The neuropathology of temporal lobe epilepsy. Oxford University Press, UK.

7. Berkovic SF, Andermann F, Olivier A, Ethier R, Melanson D, et al. 1991. Hippocampal sclerosis in temporal lobe epilepsy demonstrated by magnetic resonance imaging. Ann Neurol 29(2): 175-182. https:// doi.org/10.1002/ana.410290210

8. Jackson G, Berkovic S, Tress B, Kalnins R, Fabinyi G, et al. 1990. Hippocampal sclerosis can be reliably detected by magnetic resonance imaging. Neurology 40(12): 1869-1875. https://doi.org/10.1212/ WNL.40.12.1869

9. Gates JR, Cruz-Rodriguez R. 1990. Mesial temporal sclerosis: pathogenesis, diagnosis, and management. Epilepsia 31(Suppl 3): S55-S66. https://doi.org/10.1111/j.1528-1157.1990.tb05860.x

10. den Heijer T, Sijens P, Prins N, Hofman A, Koudstaal P, et al. 2006. MR spectroscopy of brain white matter in the prediction of dementia. Neurology 66(4): 540-544. https://doi.org/10.1212/01. wnl.0000198256.54809.0e

11. Metastasio A, Rinaldi P, Tarducci R, Mariani E, Feliziani FT, et al. 2006. Conversion of MCI to dementia: role of proton magnetic resonance spectroscopy. Neurobiol Aging 27(7): 926-932. https://doi. org/10.1016/j.neurobiolaging.2005.05.002

12. Kantarci K, Reynolds G, Petersen RC, Boeve BF, Knopman DS, et al. 2003. Proton MR spectroscopy in mild cognitive impairment and Alzheimer disease: comparison of 1.5 and 3T. AJNR Am J Neuroradiol 24(5): 843-849.

13. Kantarci K, Smith GE, Ivnik RJ, Petersen RC, Boeve BF, et al. 2002. ${ }^{1} \mathrm{H}$ magnetic resonance spectroscopy, cognitive function, and apolipoprotein $\mathrm{E}$ genotype in normal aging, mild cognitive impairment and Alzheimer's disease. J Int Neuropsychol Soc 8(7): 934-942. https:// doi.org/10.1017/S1355617702870084

14. Kantarci K, Weigand SD, Petersen RC, Boeve BF, Knopman DS, et al. 2007. Longitudinal ${ }^{1} \mathrm{H}$ MRS changes in mild cognitive impairment and Alzheimer's disease. Neurobiol Aging 28(9): 1330-1339. https://doi. org/10.1016/j.neurobiolaging.2006.06.018

15. Catani M, Cherubini A, Howard R, Tarducci R, Pelliccioli G, et al. 2001. ${ }^{1} \mathrm{H}-\mathrm{MR}$ spectroscopy differentiates mild cognitive impairment from normal brain aging. Neuroreport 12(11): 2315-2317.

16. Doraiswamy PM, Charles HC, Krishnan KRR. 1998. Prediction of cognitive decline in early Alzheimer's disease. The Lancet 352(9141): 1678. https://doi.org/10.1016/S0140-6736(05)61449-3

17. Suhy J, Miller R, Rule R, Schuff N, Licht J, et al. 2002. Early detection and longitudinal changes in amyotrophic lateral sclerosis by ${ }^{1} \mathrm{H}$ MRSI Neurology 58(5): 773-779.

18. Schott JM, Frost C, MacManus DG, Ibrahim F, Waldman AD, et al. 2010. Short echo time proton magnetic resonance spectroscopy in Alzheimer's disease: a longitudinal multiple time point study. Brain 133(11): 3315-3322. https://doi.org/10.1093/brain/awq208

19. Laprie A, Pirzkall A, Haas-Kogan DA, Cha S, Banerjee A, et al. 2005. Longitudinal multivoxel MR spectroscopy study of pediatric diffuse brainstem gliomas treated with radiotherapy. Int J Radiat Oncol Biol Phys 62(1): 20-31. https://doi.org/10.1016/j.ijrobp.2004.09.027

20. Davanzo P, Thomas MA, Yue K, Oshiro T, Belin T, et al. 2001. Decreased anterior cingulate myo-inositol/creatine spectroscopy resonance with lithium treatment in children with bipolar disorder. Neuropsychopharmacology 24(4): 359-369. https://doi.org/10.1016/ S0893-133X(00)00207-4

21. Bottomley PA. 1991. The trouble with spectroscopy papers. Radiology 181(2): 344-350. https://doi.org/10.1148/radiology.181.2.1924769

22. Kreis R. 2004. Issues of spectral quality in clinical ${ }^{1} \mathrm{H}$-magnetic resonance spectroscopy and a gallery of artifacts. NMR Biomed 17(6): 361-381. https://doi.org/10.1002/nbm.891
23. Erecińska M, Silver IA. 1990. Metabolism and role of glutamate in mammalian brain. Prog Neurobiol 35(4): 245-296. https://doi. org/10.1016/0301-0082(90)90013-7

24. Pouwels PJ, Frahm J. 1998. Regional metabolite concentrations in human brain as determined by quantitative localized proton MRS. Magn Reson Med 39(1): 53-60. https://doi.org/10.1002/mrm.1910390110

25. Block W, Träber F, Kuhl C, Fric M, Keller E, et al. 1995. ${ }^{1} \mathrm{H}-\mathrm{MR}$ spektroskopische Bildgebung bei Patienten mit klinisch gesichertem Morbus Alzheimer. In: RÖFO. Fortschritte auf dem Gebiete der Röntgenstrahlen und der neuen bildgebenden Verfahren, Thieme, pp 230-237.

26. O’Neill J, Schuff N, Marks WJ, Feiwell R, Aminoff MJ, et al. 2002. Quantitative ${ }^{1} \mathrm{H}$ magnetic resonance spectroscopy and MRI of Parkinson's disease. Mov Disord 17(5): 917-927. https://doi. org/10.1002/mds.10214

27. Brex P, Parker G, Leary S, Molyneux P, Barker G, et al. 2000. Lesion heterogeneity in multiple sclerosis: a study of the relations between appearances on T1 weighted images, T1 relaxation times, and metabolite concentrations. J Neurol Neurosurg Psychiatry 68(5): 627-632. https:// doi.org/10.1136/jnnp.68.5.627

28. Dou W, Speck O, Benner T, Kaufmann J, Li M, et al. 2015. Automatic voxel positioning for MRS at 7 T. MAGMA 28(3): 259-270. https://doi. org/10.1007/s10334-014-0469-9

29. Storrs J. 2010. Automatic Real-Time Targeting of Single-Voxel Magnetic Resonance Spectroscopy. University of Cincinnati, OH, USA.

30. Hancu I, Blezek DJ, Dumoulin MC. 2005. Automatic repositioning of single voxels in longitudinal ${ }^{1} \mathrm{H}$ MRS studies. NMR Biomed 18(6): 352361. https://doi.org/10.1002/nbm.965

31. Ratai EM, Hancu I, Blezek DJ, Turk KW, Halpern E, et al. 2008. Automatic repositioning of MRSI voxels in longitudinal studies: impact on reproducibility of metabolite concentration measurements. J Magn Reson Imaging 27(5): 1188-1193. https://doi.org/10.1002/jmri.21365

32. Jenkinson M, Smith S. 2001. A global optimisation method for robust affine registration of brain images. Med Image Anal 5(2): 143-156. https://doi.org/10.1016/S1361-8415(01)00036-6

33. Bai X, Harris AD, Gong T, Puts NA, Wang G, et al. 2017. Voxel placement precision for GABA-edited magnetic resonance spectroscopy. Open J Radiol 7(1): 35-44. https://doi.org/10.4236/ojrad.2017.71004

34. Lee H, Caparelli E, Li H, Mandal A, Smith SD, et al. 2013. Computerized MRS voxel registration and partial volume effects in single voxel ${ }^{1} \mathrm{H}-\mathrm{MRS}$. Magn Reson Imaging 31(7): 1197-1205. https:// doi.org/10.1016/j.mri.2013.04.001

35. van der Kouwe AJ, Benner T, Fischl B, Schmitt F, Salat DH, et al. 2005. On-line automatic slice positioning for brain MR imaging. NeuroImage 27(1): 222-230. https://doi.org/10.1016/j.neuroimage.2005.03.035

36. Gedat E, Braun J, Sack I, Bernarding J. 2004. Prospective registration of human head magnetic resonance images for reproducible slice positioning using localizer images. J Magn Reson Imaging 20(4): 581587. https://doi.org/10.1002/jmri.20153

37. Welch EB, Manduca A, Grimm RC, Jack CR. 2004. Interscan registration using navigator echoes. Magn reson Med 52(6): 1448-1452. https://doi.org/10.1002/mrm.20275

38. Itti L, Chang L, Ernst T. 2001. Automatic scan prescription for brain MRI. Magn Reson Med 45(3): 486-494. https://doi.org/10.1002/15222594(200103)45:3<486::AID-MRM1064>3.0.CO;2-\#

39. Hartmann SL, Dawant BM, Parks MH, Schlack H, Martin PR. 1998. Image-guided MR spectroscopy volume of interest localization for longitudinal studies. Comput Med Imaging Graph 22(6): 453-461. https://doi.org/10.1016/S0895-6111(98)00050-0

40. Benner T, Wisco JJ, van der Kouwe AJ, Fischl B, Vangel MG, et al. 2006. Comparison of Manual and automatic section positioning of brain MR images. Radiology 239(1): 246-254. https://doi.org/10.1148/ radiol.2391050221 